\title{
RhoBAST - a rhodamine-binding aptamer for super-resolution RNA imaging
}

Murat Sunbul ${ }^{1 *}$, Jens Lackner², Annabell Martin¹, Daniel Englert ${ }^{1}$, Benjamin Hacene ${ }^{2}$, Karin Nienhaus $^{2}$, G. Ulrich Nienhaus ${ }^{2,3,4,5}$, and Andres Jäschke ${ }^{1 *}$

\footnotetext{
${ }^{1}$ Institute of Pharmacy and Molecular Biotechnology (IPMB), Heidelberg University, D-69120 Heidelberg, Germany

${ }^{2}$ Institute of Applied Physics (APH), Karlsruhe Institute of Technology (KIT), Wolfgang-Gaede-Str. 1, D-76131

Karlsruhe, Germany

${ }^{3}$ Institute of Nanotechnology (INT), Karlsruhe Institute of Technology (KIT), D-76344 Eggenstein-Leopoldshafen, Germany

${ }^{4}$ Institute of Biological and Chemical Systems (IBCS), Karlsruhe Institute of Technology (KIT), D-76344

Eggenstein-Leopoldshafen, Germany

${ }^{5}$ Department of Physics, University of Illinois at Urbana-Champaign, 1110 West Green Street, Urbana, Illinois 61801, United States
}

\begin{abstract}
RhoBAST is a novel fluorescence light-up RNA aptamer (FLAP) that transiently binds a fluorogenic rhodamine dye. Fast dye association and dissociation result in intermittent fluorescence emission, facilitating single-molecule localization microscopy (SMLM) with an image resolution not limited by photobleaching. We demonstrate RhoBAST's excellent properties as a RNA marker by resolving subcellular and subnuclear structures of RNA in live and fixed cells by SMLM and structured illumination microscopy (SIM).
\end{abstract}




\section{Main}

Super-resolution fluorescence microscopy of cellular structures continually provides fundamentally new insights into biology ${ }^{1}$. Over the years, an elaborate toolbox has become available to attach fluorescent markers to proteins for such studies. By contrast, marker tools for super-resolution RNA imaging are still scarce $^{2}$. Recently, FLAPs have been recognized as promising tools for RNA imaging ${ }^{3}$. So far, they have mostly been employed for conventional, diffraction-limited imaging. FLAPs for super-resolution imaging have been reported only last year, specifically, the SiRA aptamer ${ }^{4}$ for stimulated emission depletion (STED) microscopy and the Pepper aptamer $^{5}$ for structured illumination microscopy (SIM). Further optimization of these RNA markers will facilitate their widespread use in super-resolution microscopy.

Our laboratory has developed FLAPs that bind fluorophore-quencher conjugates and thereby disrupt contact quenching (Fig. 1a $)^{6,7}$. This strategy affords the use of bright and photostable state-of-theart fluorophores for RNA imaging ${ }^{7,8}$. For example, the SRB-2 aptamer binds various membrane-permeable rhodamine dyes with high affinity, yet it does not provide sufficient sensitivity for imaging RNAs of low abundance in cells ${ }^{9}$. Here we present RhoBAST, a FLAP endowed with excellent properties for singlemolecule localization microscopy $(\mathrm{SMLM})^{10}$, and demonstrate its ability to resolve subnuclear structures of RNA in live and fixed cells using SMLM and SIM.

Starting from a 15\% doped SRB-2 aptamer library, in vitro evolution was employed to improve the affinity, thermal stability, photostability and brightness of SRB-2 using tetramethylrhodamine (TMR) as bait (Fig. 1b,c). Following incubation of the library with TMR-decorated beads, mutants binding TMR were specifically eluted, amplified and subsequently used as input for the next round of evolution. After seven iterations (Fig. 1d), the enriched variants were sequenced and screened for fluorescence enhancement upon binding the dye-quencher conjugate TMR-dinitroaniline (TMR-DN, Supplementary Fig. 1). Sequence and truncation analysis (Supplementary Figs. 2 and 3) revealed two beneficial mutations in the SRB-2 sequence, namely an insertion of a single $U$ nucleotide between $C 6$ and $U 7$ and a U22A mutation (Supplementary Fig. 4). These features were incorporated into the design of SRB-3 (Supplementary Fig. 4), which exhibits $50 \%$ higher brightness and 1.8-fold higher affinity $\left(K_{D}=20 \pm 1 \mathrm{nM}\right)$ for TMR-DN than SRB-2 (Fig. 1f). 
For a FLAP to function in a biological environment, folding into a unique fluorophore-binding structure is essential, as alternative folds would lead to lower sensitivity. To eliminate alternative secondary structures predicted for SRB-3 (Supplementary Fig. 5), we rationally exchanged the U8-A19 base pair by C-G and the UUCG tetraloop by GAAA (Fig. 1e). Indeed, these mutations were beneficial, resulting in a new aptamer dubbed RhoBAST (Rhodamine Binding Aptamer for Super-resolution Imaging Techniques). It features a fraction of $86 \pm 5 \%$ in the proper fold, a 26 -fold fluorescence turn-on and a $K_{\mathrm{D}}$ of $15 \pm 1 \mathrm{nM}$ (Fig. 1f).

SRB-2, SRB-3 and RhoBAST have similar circular dichroism (CD) spectra (Supplementary Fig. 6). The fluorescence emission of their complexes with TMR-DN is independent of sodium or potassium ions but strictly depends on the magnesium ion $\left(\mathrm{Mg}^{2+}\right)$ concentration (Supplementary Fig. 7), in contrast to Gquadruplex-forming FLAPs ${ }^{11-13}$. Yet, RhoBAST:TMR-DN retains more than $90 \%$ of its maximum fluorescence at $0.25 \mathrm{mM} \mathrm{Mg}^{2+}$, implying that RhoBAST functions effectively under physiological conditions (Supplementary Fig. 7). RhoBAST:TMR-DN exhibits excellent brightness and well-defined fluorescence spectra, with excitation and emission maxima at 564 and $590 \mathrm{~nm}$, respectively (Fig. $1 \mathrm{~g}, \mathrm{~h}$ ). It is 4.6- and 7.6-fold brighter than Broccoli:DFHBI-1T ${ }^{11}$ and Corn:DFHO ${ }^{12}$, respectively (Supplementary Table 1). With a melting point, $\mathrm{T}_{\mathrm{m}}$, of $79{ }^{\circ} \mathrm{C}$, RhoBAST is extremely thermostable. Consequently, upon raising the temperature to $37^{\circ} \mathrm{C}$, RhoBAST:TMR-DN retains $69 \%$ of its fluorescence intensity at $25^{\circ} \mathrm{C}$, whereas it drops to $54 \%$ for SRB-2:TMR-DN (Supplementary Fig. 6). Furthermore, RhoBAST:TMR-DN appears remarkably photostable; its fluorescence only decreased by about $1 \%$ over $3 \mathrm{~h}$ under continuous illumination, whereas $12 \%$ of the rhodamine-based $\mathrm{JF}_{549}$-Halo fluorophore and $50 \%$ of the mRuby3 fluorescent protein were photobleached under identical conditions (Fig. 1i). Taken together, these favorable characteristics make the RhoBAST:TMR-DN system a superb genetically encoded tag to image RNA in live cells.

Next, we evaluated how RhoBAST performs in E. coli cells in comparison to SRB-2 and SRB-3. Single copies of the aptamers without stabilizing scaffolds were fused to the $3^{\prime}$ untranslated region (UTR) of $g f p$ (Fig. 2a). In the TMR channel of confocal images, the signal-to-background (S/B) ratios for bacteria expressing gfp-RhoBAST, gfp-SRB-3 and gfp-SRB-2 mRNAs were, respectively, $14 \pm 1,8 \pm 1$ and $4 \pm 1$. $\mathrm{S} / \mathrm{B}$ ratios were calculated as the ratio of the fluorescence intensity (mean \pm s.e.m.) at the poles of bacteria 
expressing gfp-aptamer to that of bacteria expressing only gfp mRNA (Fig. 2a,b). Moreover, the fluorescence intensity of multiple RhoBAST repeats increased almost linearly up to 16 (Supplementary Fig. 8), which enables imaging of low-abundance RNAs. To analyze the sensitivity of RhoBAST:TMR-DN, bacteria expressing different amounts of $g f p-R h o B A S T_{16}$ mRNA were fixed with paraformaldehyde and imaged. Even at a level of $\sim 1$ molecule of gfp-RhoBAST 16 per cell (quantified by RT-qPCR), significantly higher fluorescence was detected in the TMR channel than in control bacteria expressing only gfp mRNA (Supplementary Fig. 8). Furthermore, to image endogenously expressed mRNAs, multiple RhoBAST repeats were stably introduced into the genome of $E$. coli $\mathrm{K} 12$, and $o m p A$, rnase $E$ and cat mRNAs were visualized displaying distinct localization patterns (Fig. 2c).

To investigate the applicability of RhoBAST:TMR-DN to the imaging of live mammalian cells, a diverse set of RNA species with different reported localization patterns and transcribed from different promoters were examined. First, RhoBAST embedded into the Tornado plasmid system ${ }^{14}$ was expressed as a single-copy, circular aptamer under the control of the U6 promoter in HEK293T cells. Besides nuclear puncta, most of the circular RhoBAST was observed in the cytosol (Fig. 2d), as previously reported for circular Broccoli ${ }^{14}$ and Corn ${ }^{15}$. This demonstrates that RhoBAST folds properly and functions as expected in mammalian cells. Second, GFP mRNA tagged with RhoBAST ${ }_{16}$ was expressed under the CAG promoter in live HEK293T cells, revealing the typical cytosolic localization of GFP mRNA (Fig. 2e). Third, the CGG trinucleotide repeat-containing FMR1 (fragile-X mental retardation gene)-GFP mRNA fused to RhoBAST 16 was expressed in HeLa cells under the CMV promoter. It showed the distinct nuclear aggregation pattern reported for this $\mathrm{mRNA}^{16}$ (Fig. 2f). The low fluorescence in the TMR channel of control cells demonstrates the specific effect of RhoBAST on the TMR-DN fluorescence (Fig. 2g).

RhoBAST:TMR-DN appears well-suited for $\mathrm{SIM}^{17}$ owing to its remarkable photostability and brightness. We examined its performance for SIM by imaging tolB-gfp-RhoBAST 16 mRNA in bacteria. The TolB signal peptide causes localization of the mRNA at the inner bacterial membrane due to signal recognition particle (SRP) mediated co-translational translocation ${ }^{18}$ of TolB-GFP. SIM images clearly revealed that $t o l B-g f p-R h o B A S T_{16}$ mRNA localized at the inner membrane, whereas $g f p-R h o B A S T_{16}$ lacking the tolB sequence or carrying another SRP-independent signal peptide sequence showed substantially different localizations (Supplementary Fig. 9). 
Next, we examined the compatibility of RhoBAST:TMR-DN with SMLM by measuring the kinetic parameters of TMR-DN association to and dissociation from the aptamer, generating intermittent fluorescence emission, which is essential for SMLM. To this end, RhoBAST molecules were sparsely immobilized on glass surfaces (Fig. 3a) and, after adding TMR-DN solution, fluorescence intensity time traces from individual molecules were acquired (Fig. 3b). Analysis of the fluorescence on and off times in these traces as a function of the TMR-DN concentration in the range of $1-10 \mathrm{nM}$ yields dissociation $\left(k_{d}\right.$ $\left.=0.66 \pm 0.01 \mathrm{~s}^{-1}\right)$ and association $\left(k_{\mathrm{a}}=1.8 \pm 0.1 \times 10^{7} \mathrm{M}^{-1} \mathrm{~s}^{-1}\right)$ rate coefficients (Fig. 3b,c). These values indicate that a TMR-DN ligand remains bound to RhoBAST for $1.5 \mathrm{~s}$, dissociates and is replaced by a new ligand within about $5 \mathrm{~s}$ (at $10 \mathrm{nM}$ ). Thus, RhoBAST features a more than two orders of magnitude higher blinking frequency than other FLAPs (Supplementary Table 2). Due to continuous ligand exchange, the number of photons captured from the aptamer is not limited by photobleaching. As a consequence, molecular positions can be determined precisely, as in DNA-PAINT ${ }^{19}$.

To assess RhoBAST:TMR-DN in SMLM applications, we visualized structures inside the cell nucleus formed upon expression of circular RhoBAST in live and fixed HEK293T cells. Intriguingly, we observed hollow spheres in the nucleus, details of which are hardly visible in the epifluorescence image (Fig. 3d, Supplementary Fig. 10). Elaborate immunofluorescence and colocalization studies of these subnuclear structures with literature-reported nuclear bodies (Supplementary Fig. 11) revealed that cyclic RhoBAST in the nucleus co-localized with NONO and PSPC1, proteins that are crucial components of paraspeckle nuclear bodies ${ }^{20}$. Presumably, the high concentration of circular RhoBAST in the nucleus triggers phase separation of RNA with the assistance of NONO and PSPC1, forming paraspeckle-like nuclear bodies (Supplementary Fig. 12).

Multiple repeats of RhoBAST can be introduced to increase the total number of association and dissociation events within a given time interval. Thus, the overall image acquisition time decreases, as photons can be collected at a higher rate to pinpoint the position of the target RNA, albeit with somewhat lower spatial resolution due to the larger tag size. Indeed, rapidly acquired SMLM images of RhoBAST ${ }_{16}^{-}$ tagged gfp, tolB-gfp and phoA-gfp mRNAs show the correct localization patterns (Supplementary Fig. 1314), and images reconstructed from 5,000 camera frames (Fig. 3f,g) confirm the excellent image quality. 
Here we have introduced a next generation rhodamine-binding aptamer, RhoBAST, as a genetically encoded tag for super-resolution RNA imaging. In comparison to its parent SRB-2, it features markedly improved folding and thermal stability, higher affinity to TMR-DN as well as higher fluorescence quantum yield and photobleaching resistance. Taken together, these advances result in RhoBAST's excellent performance as an RNA marker for fluorescence imaging. The RhoBAST:TMR-DN system is the first example of a FLAP that is well suited for live-cell SMLM, owing to a continuous and fast fluorophore exchange combined with exceptionally high photostability and brightness. With RhoBAST:TMR-DN, subcellular and subnuclear structures of RNA can readily be visualized with high localization precision in live or fixed specimens.

\section{References}

1. Sigal, Y.M., Zhou, R. \& Zhuang, X. Visualizing and discovering cellular structures with super-resolution microscopy. Science 361, 880-887 (2018).

2. Schmidt, A., Gao, G., Little, S.R., Jalihal, A.P. \& Walter, N.G. Following the messenger: Recent innovations in live cell single molecule fluorescence imaging. Wiley Interdiscip Rev RNA, e1587 (2020).

3. Su, Y. \& Hammond, M.C. RNA-based fluorescent biosensors for live cell imaging of small molecules and RNAs. Curr Opin Biotechnol 63, 157-166 (2020).

4. Wirth, R., Gao, P., Nienhaus, G.U., Sunbul, M. \& Jäschke, A. SiRA: A Silicon Rhodamine-Binding Aptamer for Live-Cell Super-Resolution RNA Imaging. J Am Chem Soc 141, 7562 (2019).

5. Chen, $X$. et al. Visualizing RNA dynamics in live cells with bright and stable fluorescent RNAs. Nat Biotechnol 37, 1287-1293 (2019).

6. Sunbul, M. \& Jäschke, A. Contact-mediated quenching for RNA imaging in bacteria with a fluorophore-binding aptamer. Angew Chem, Int Ed 52, 13401-13404 (2013).

7. Arora, A., Sunbul, M. \& Jäschke, A. Dual-colour imaging of RNAs using quencher- and fluorophore-binding aptamers. Nucleic Acids Res 43, e144 (2015).

8. Braselmann, E. et al. A multicolor riboswitch-based platform for imaging of RNA in live mammalian cells. Nat Chem Biol 14, 964-971 (2018).

9. Sunbul, M. \& Jaschke, A. SRB-2: a promiscuous rainbow aptamer for live-cell RNA imaging. Nucleic Acids Res 46, e110 (2018).

10. Li, Y., Ishitsuka, Y., Hedde, P.N. \& Nienhaus, G.U. Fast and efficient molecule detection in localization-based super-resolution microscopy by parallel adaptive histogram equalization. ACS Nano 7, 5207-5214 (2013).

11. Filonov, G.S., Moon, J.D., Svensen, N. \& Jaffrey, S.R. Broccoli: rapid selection of an RNA mimic of green fluorescent protein by fluorescence-based selection and directed evolution. J Am Chem Soc 136, 16299-16308 (2014).

12. Song, W. et al. Imaging RNA polymerase III transcription using a photostable RNA-fluorophore complex. Nat Chem Biol 13, 1187-1194 (2017).

13. Autour, A. et al. Fluorogenic RNA Mango aptamers for imaging small non-coding RNAs in mammalian cells. Nat Commun 9, 656 (2018).

14. Litke, J.L. \& Jaffrey, S.R. Highly efficient expression of circular RNA aptamers in cells using autocatalytic transcripts. Nat Biotechnol 37, 667-675 (2019). 
15. Kim, H. \& Jaffrey, S.R. A Fluorogenic RNA-Based Sensor Activated by Metabolite-Induced RNA Dimerization. Cell Chem Biol 26, 1725-1731 e1726 (2019).

16. Hagerman, R.J. \& Hagerman, P. Fragile X-associated tremor/ataxia syndrome - features, mechanisms and management. Nat Rev Neurol 12, 403-412 (2016).

17. $\mathrm{Wu}, \mathrm{Y}$. \& Shroff, H. Faster, sharper, and deeper: structured illumination microscopy for biological imaging. Nat Methods 15, 1011-1019 (2018).

18. Steinberg, R., Knupffer, L., Origi, A., Asti, R. \& Koch, H.G. Co-translational protein targeting in bacteria. FEMS Microbiol Lett 365 (2018).

19. Schnitzbauer, J., Strauss, M.T., Schlichthaerle, T., Schueder, F. \& Jungmann, R. Super-resolution microscopy with DNA-PAINT. Nature protocols 12, 1198-1228 (2017).

20. Fox, A.H., Nakagawa, S., Hirose, T. \& Bond, C.S. Paraspeckles: Where Long Noncoding RNA Meets Phase Separation. Trends Biochem Sci 43, 124-135 (2018). 


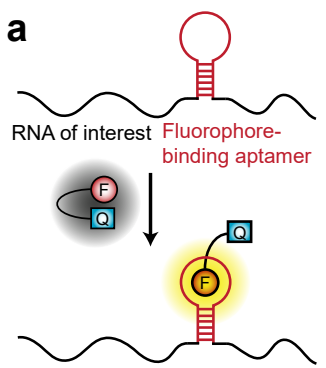

e

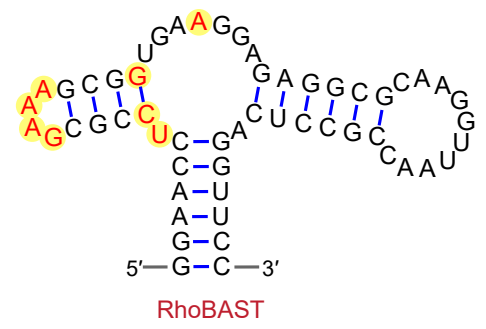

g

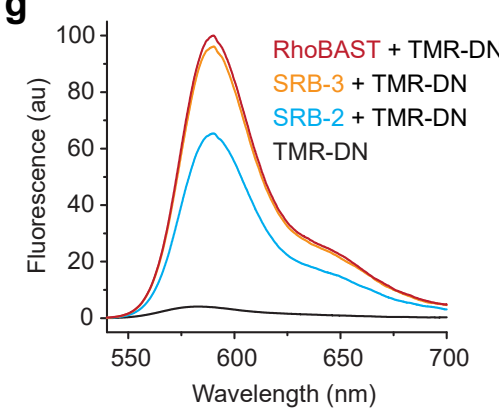

b

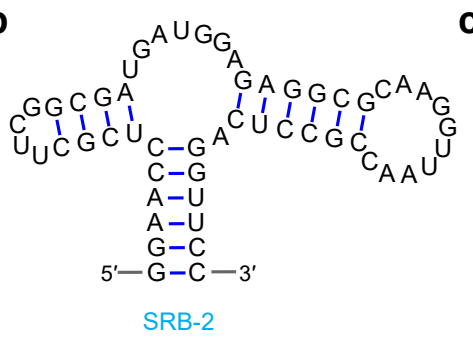

f

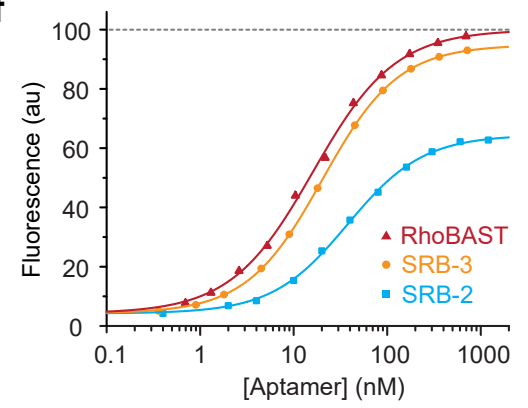

h

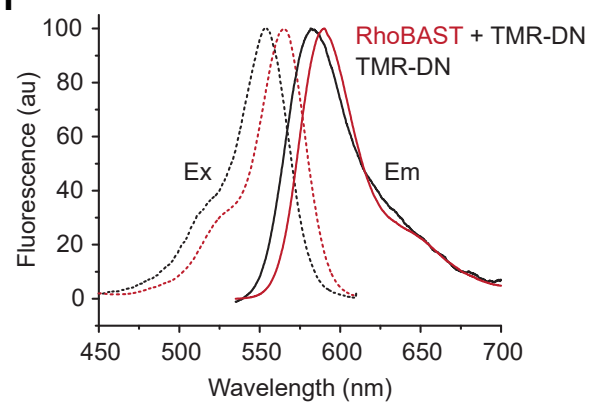

d
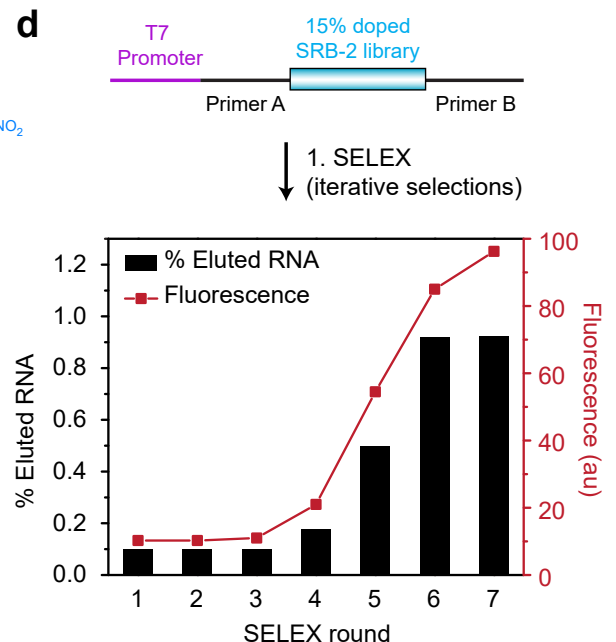

2. Sequence analysis \& aptamer characterization

3. Rational design \&

$\downarrow$ aptamer characterization

RhoBAST

i

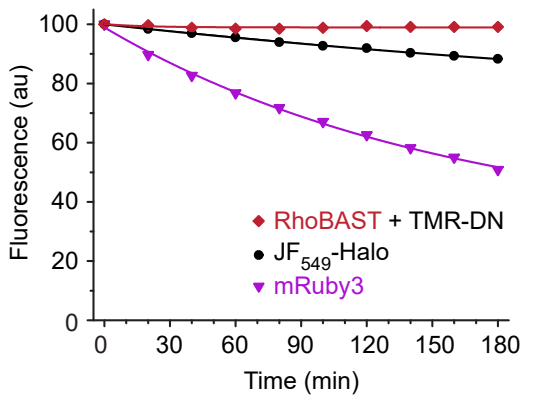

Figure 1. Directed evolution and characterization of RhoBAST. a) Scheme showing the use of a genetically encoded fluorophore-binding aptamer and a contact-quenched fluorophore-quencher conjugate (F-Q) for imaging an RNA of interest. $F-Q$ is non-fluorescent in solution and lights up upon binding to the aptamer. b) Predicted secondary structure of SRB-2. c) Chemical structure of TMR-DN. d) Directed evolution of RhoBAST for binding to the TMR fluorophore using iterative cycles of SELEX and rational design. The initial library was composed of 15\% doped SRB-2 flanked by constant primer $A$ and primer B binding regions. The T7 promoter was used for efficient transcription of the library by T7 RNA polymerase. Progress of the selection was assessed by calculating the percentage of eluted RNA and the fluorescence enhancement of TMR-DN upon addition of RNA pools after each round. e) Predicted secondary structure of RhoBAST; changed ribonucleotides are highlighted in yellow. $\mathbf{f})$ Binding isotherms resulting in equilibrium dissociation coefficients, $K_{\mathrm{D}}$, of $35 \pm 1 \mathrm{nM}$ for SRB-2, $20 \pm 1 \mathrm{nM}$ for SRB-3 and 15 $\pm 1 \mathrm{nM}$ for RhoBAST, using TMR-DN as the ligand. g) Emission spectra of TMR-DN, SRB-2:TMR-DN, SRB-3:TMR-DN and RhoBAST:TMR-DN, all measured under identical conditions and normalized such that the RhoBAST:TMR-DN spectrum peaks at 100. h) Normalized excitation and emission spectra of TMR-DN and RhoBAST:TMR-DN. i) Fluorescence intensity decay of TMR-DN (20 nM) complexed with RhoBAST (500 nM), the $\mathrm{JF}_{549}$-Halo fluorophore $(20 \mathrm{nM})$ and the mRuby3 fluorescent protein (20 nM). After $3 \mathrm{~h}$, the fractional decrease was $0.9 \pm 0.4 \%, 11.7 \pm 0.9 \%$ and $49.5 \pm 2.7 \%$ (mean \pm s.d.), respectively. 
a

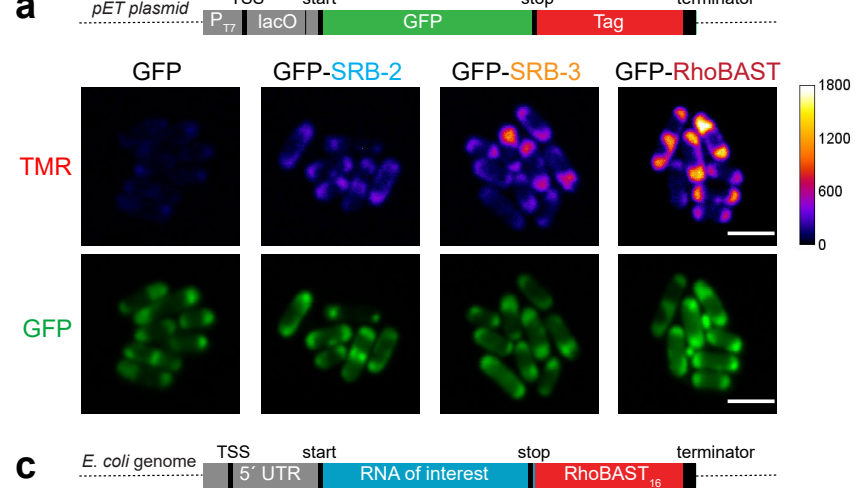

b

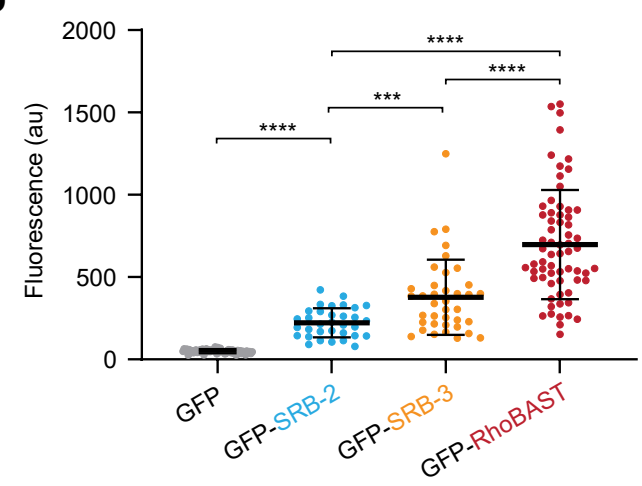

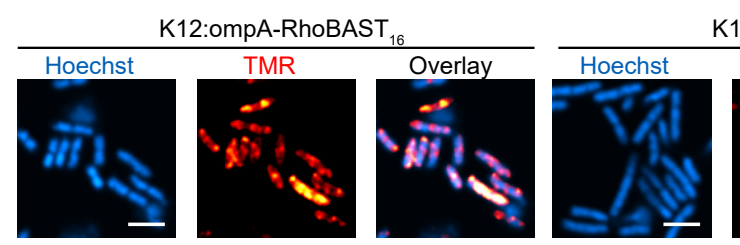

d

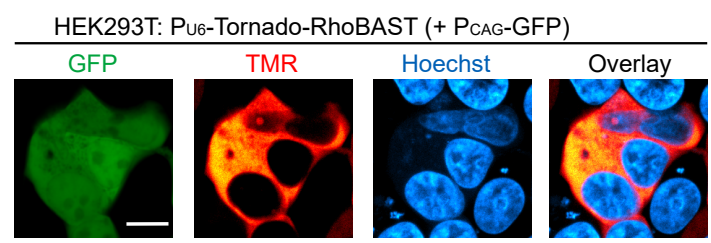

e HEK293T: PCAG-GFP-RhoBAST 16

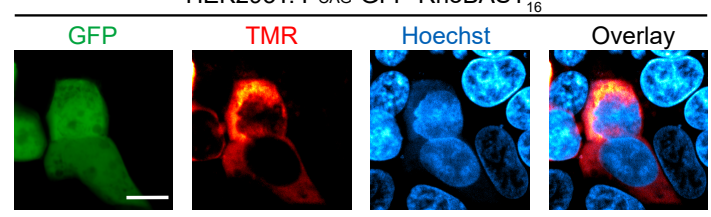

K12:cat-RhoBAST

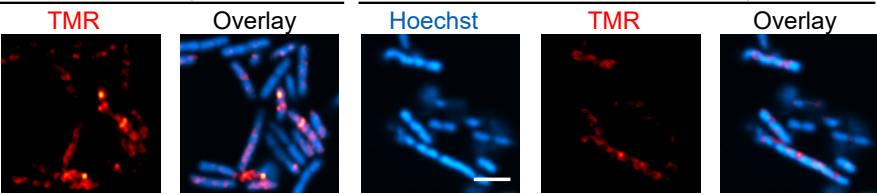

f

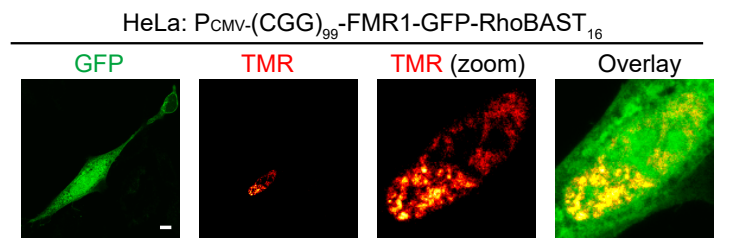

g

HEK293T: PCAG-GFP (control)

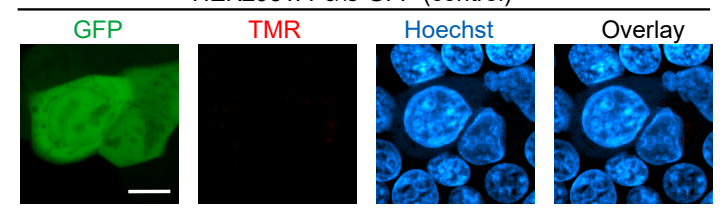

Figure 2. Performance of RhoBAST as a marker for RNA imaging in live cells. a) Live cell imaging of gfp mRNA (control) and gfp mRNA fused to a single copy of SRB-2, SRB-3 or RhoBAST. Bacteria were transformed with the pET plasmid carrying the gfp gene fused to the aptamer tags at the 3' UTR. Scale bars, $3 \mu \mathrm{m}$. b) Quantification of the average TMR fluorescence at the poles of bacteria expressing the different RNA constructs. Each dot represents a single cell; also indicated are the means \pm s.d. ( $N>30$ cells). Statistical comparison was performed by using a two-tailed t-test. ${ }^{* * *}, P \leq 0.001 ;{ }^{* * *}, P \leq 0.0001$. c) Live-cell mRNA imaging of endogenously RhoBAST-tagged ompA, cat and rnaseE in $E$. coli K12. d) Live-cell imaging of circular RhoBAST aptamers transcribed under the control of the U6 promoter using the Tornado system in HEK293T cells. Cells were cotransfected with another GFP-expressing plasmid as a transfection control. e) Live-cell imaging of GFP mRNA fused to RhoBAST 16 transcribed under the CAG promoter in HEK293T cells. f) Live-cell imaging of the trinucleotide CGG repeat-containing FMR1-GFP mRNA fused to RhoBAST ${ }_{16}$ transcribed under the CMV promoter in HeLa cells. g) Live-cell imaging of HEK293T cells transfected with a GFP expressing plasmid (negative control) to show the background fluorescence due to the presence of free TMR-DN. At least three independent experiments were carried out with similar results. 
a

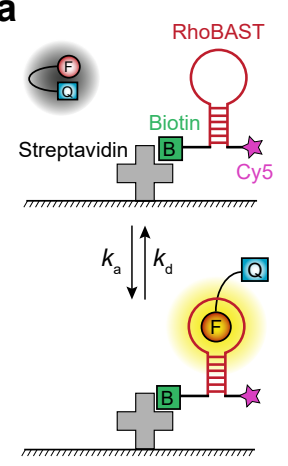

d TMR (epifluorescence)

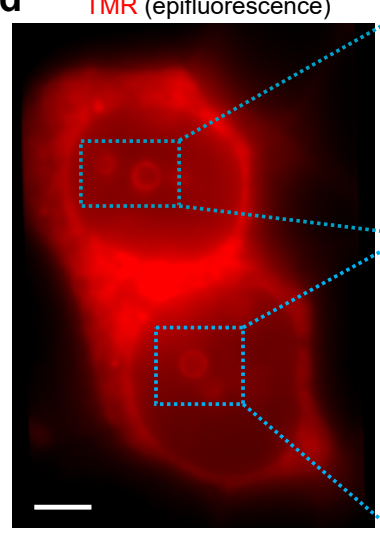

f

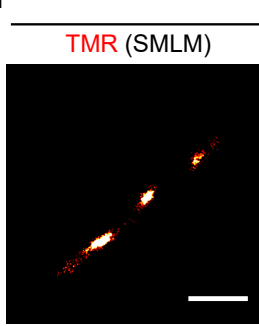

b
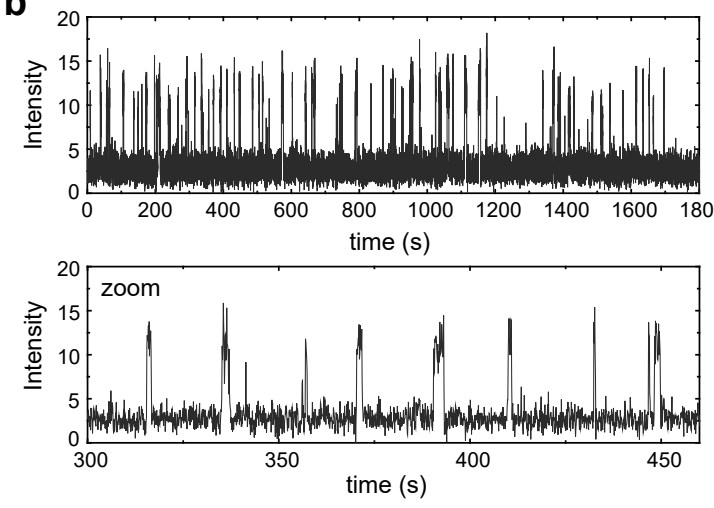

Epifluorescence

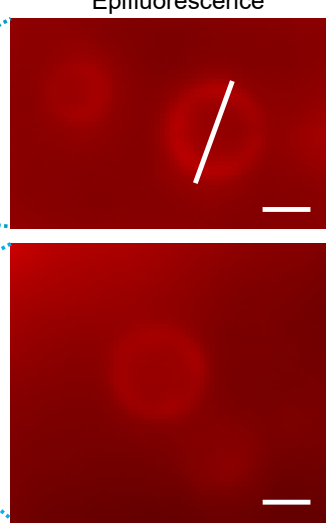

TolB-GFP-RhoBAST ${ }_{16}$
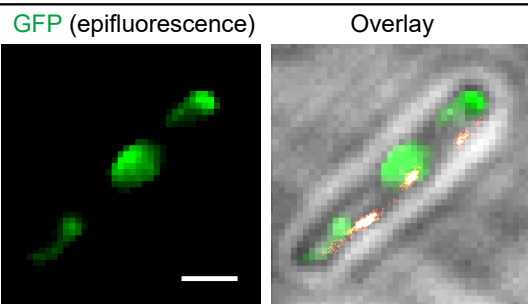

SMLM

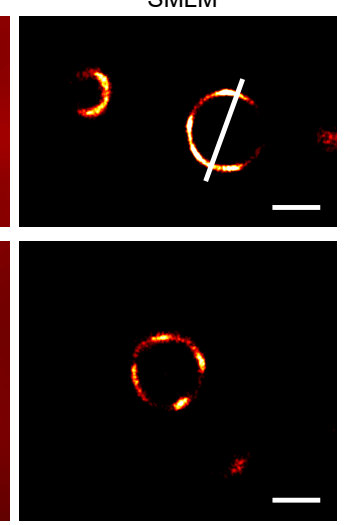

g

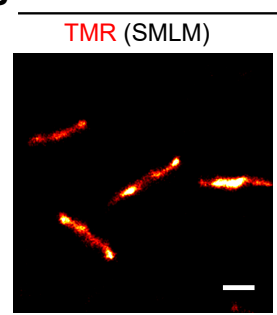

e
C
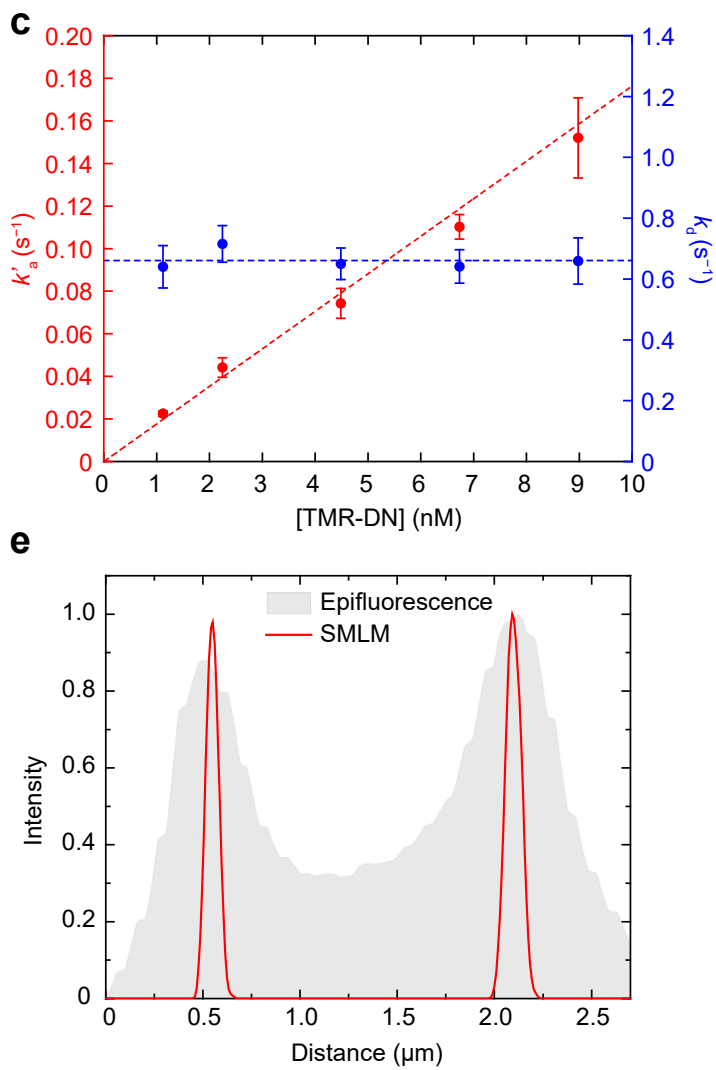

GFP-RhoBAST $_{16}$

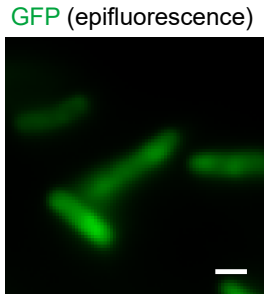

Overlay

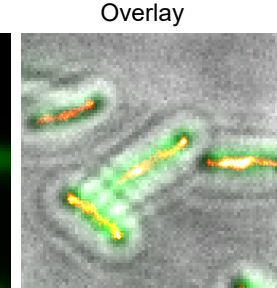

Figure 3. SMLM with the RhOBAST:TMR-DN system. a) Scheme showing on and off switching of the fluorescence due to TMR-DN association to and dissociation from RhoBAST immobilized on a cover slip. RhoBAST was modified with biotin at $5^{\prime}$ and Cy 5 at $3^{\prime}$ and bound to the glass surface via streptavidin. b) Fluorescence time trace taken on an individual, surface-immobilized RhoBAST molecule after addition of TMR-DN (2.2 nM) containing buffer. It reveals intermittent fluorescence emission due to continuous TMRDN association and dissociation. c) Dependence of the average dissociation $\left(k_{d}=\left\langle T_{0 n}\right\rangle^{-1}\right)$ and association $\left(k_{\mathrm{a}}^{\prime}=k_{\mathrm{a}} c=\left\langle\mathrm{T}_{\text {off }}\right\rangle^{-1}\right)$ rate coefficients on the TMR-DN concentration (c). Here, $<T_{\mathrm{on}}>$ and $\left\langle T_{\text {off }}>\right.$ are the average times of emission and the lack thereof, respectively. Data points represent mean \pm s.d. d) Epifluorescence and SMLM images of live HEK293T cells showing hollow spheres containing circular RhoBAST in the nuclei; scale bars, 4 and $1 \mu \mathrm{m}$, respectively. e) Plot of the fluorescence intensity along the white lines in panel d, showing the much higher spatial resolution of SMLM. f) tolB-gfp and g) gfp mRNA fused to RhoBAST $_{16}$ in fixed bacteria was imaged via SMLM and epifluorescence. These images are also shown as overlays on brightfield images. At least three independent experiments were carried out with similar results. Scale bars, $1 \mu \mathrm{m}$. 


\section{Methods}

General. All reagents were purchased from Sigma-Aldrich or Thermo Fisher Scientific unless otherwise specified and used without further purification. Reverse phase HPLC purifications were performed on a Luna® $5 \mu \mathrm{m}$ C18(2) $100 \AA$ column, 250x10 mm (Phenomenex Ltd.) and compounds were eluted with a mixture of buffer $\mathrm{A}$ consisting of $100 \mathrm{mM} \mathrm{Et}_{3} \mathrm{~N} / \mathrm{AcOH}(\mathrm{pH}$ 7.0) in milli-Q-water and buffer $\mathrm{B}$ containing 100 $\mathrm{mM} \mathrm{Et}_{3} \mathrm{~N} / \mathrm{AcOH}(\mathrm{pH} 7.0)$ in an acetonitrile/water (4:1) mixture. High resolution mass spectra were recorded on a Bruker microTOFQ-II ESI mass spectrometer. DNA and RNA concentrations were determined with a NanoDrop ND-1000 spectrophotometer (NanoDrop Technologies). Fluorescence measurements were performed with a JASCO FP-6500 spectrofluorometer with an ETC-273T digital temperature controller. CD spectra were recorded on a Jasco (model J-810) spectropolarimeter. Agarose gels were stained with ethidium bromide and visualized by UV illumination using an Alphalmager ${ }^{\mathrm{TM}} 2200$ (Alpha Innotech). Absorbance spectra were recorded on a Cary 50 UV-Vis spectrophotometer (Varian). Synthetic DNA oligonucleotides were purchased from Integrated DNA Technologies (IDT). Restriction endonucleases were purchased from Thermo Fisher Scientific. HEK293T (DSMZ, ACC 635) and HeLa cells (DSMZ, ACC 57) were cultured at $37^{\circ} \mathrm{C}$ under $5 \% \mathrm{CO}_{2}$ in Dulbecco's Modified Eagle's Medium containing high glucose, HEPES and glutamine without phenol red (Thermo Fisher Scientific) supplemented with 10\% FBS, 100 unit/ml penicillin and $100 \mu \mathrm{g} / \mathrm{ml}$ streptomycin. DH5a, K12 and BL21 Star ${ }^{\mathrm{TM}}$ (DE3) cells (Thermo Fisher Scientific) were typically grown at $37^{\circ} \mathrm{C}$ with shaking at $150 \mathrm{rpm}$ in Luria-Bertani (LB) medium.

Synthesis. Synthesis and characterization of TMR-SS-Biotin (used for SELEX) and TMR-DN was explained in Supplementary Note.

DNA library preparation for SELEX. A 93-nucleotide long single-stranded DNA oligonucleotide library consisting of $15 \%$ doped 54-nucleotide SRB-2 sequence flanked by two constant primer binding sites was synthesized (IDT). DNA library sequence $\left(5^{\prime}\right.$ to $\left.3^{\prime}\right)$ :

GGAGCTCAGCCTTCACTGC(N3)(N3)(N1)(N1)(N2)(N2)(N4)(N2)(N3)(N2)(N4)(N4)(N2)(N3)(N3)(N2)(N3 )$(\mathrm{N} 1)(\mathrm{N} 4)(\mathrm{N} 3)(\mathrm{N} 1)(\mathrm{N} 4)(\mathrm{N} 3)(\mathrm{N} 3)(\mathrm{N} 1)(\mathrm{N} 3)(\mathrm{N} 1)(\mathrm{N} 3)(\mathrm{N} 3)(\mathrm{N} 2)(\mathrm{N} 3)(\mathrm{N} 2)(\mathrm{N} 1)(\mathrm{N} 1)(\mathrm{N} 3)(\mathrm{N} 3)(\mathrm{N} 4)(\mathrm{N} 4)(\mathrm{N} 1)(\mathrm{N} 1)(\mathrm{N}$ 
2)(N2)(N3)(N2)(N2)(N4)(N2)(N1)(N3)(N3)(N4)(N4)(N2)(N2)GGCACCACGGTCGGATCCAC. N1, N2, N3 and $\mathrm{N} 4$ are mixtures of $\{85 \% \mathrm{~A}, 5 \% \mathrm{C}, 5 \% \mathrm{G}, 5 \% \mathrm{~T}\},\{5 \% \mathrm{~A}, 85 \% \mathrm{C}, 5 \% \mathrm{G}, 5 \% \mathrm{~T}\},\{5 \% \mathrm{~A}, 5 \% \mathrm{C}, 85 \% \mathrm{G}$, $5 \% \mathrm{~T}\}$ and $\{5 \% \mathrm{~A}, 5 \% \mathrm{C}, 5 \% \mathrm{G}, 85 \% \mathrm{~T}\}$, respectively.

$0.5 \mathrm{nmol}$ of the oligonucleotide pool $\left(\sim 3 \times 10^{14}\right.$ molecules $)$ was amplified in a $1 \mathrm{~mL}$ PCR reaction for 8 cycles by using the forward (Primer A: 5'-TCTAATACGACTCACTATA GGAGCTCAGCCTTCACTGC-3') and reverse primers (Primer B: 5'- GTGGATCCGACCGTGGTGCC 3') to yield the double-stranded DNA template for the transcription of the library. After phenol:chloroform:isoamyl alcohol (25:24:1, pH 8) extraction, the PCR product was precipitated with sodium acetate and ethanol. The DNA pellet was dissolved in water and directly used for in vitro transcription reaction.

SELEX. For the first round of selection, a large-scale in vitro transcription mixture $(1 \mathrm{~mL})$ containing doublestranded DNA template $(0.5 \mu \mathrm{M})$, transcription buffer (40 mM Tris $\mathrm{pH} 8.1,2 \mathrm{mM}$ spermidine, $22 \mathrm{mM} \mathrm{MgCl}$, 0.01\% Triton-X-100), DTT (10 mM), BSA $(0.01 \mathrm{mg} / \mathrm{mL})$, NTP (4 mM of each ATP, CTP, GTP and UTP) and T7 RNA polymerase ( $1 \mu \mathrm{M}$, lab prepared stock) was prepared. After incubation for $4 \mathrm{~h}$ at $37^{\circ} \mathrm{C}$, DNasel (50 U) was added into the mixture and incubated for $30 \mathrm{~min}$ at $37^{\circ} \mathrm{C}$. RNA was purified on a $10 \%$ denaturing polyacrylamide gel, excised from the gel and eluted into $0.3 \mathrm{M}$ sodium acetate buffer (pH 5.5) overnight. RNA was precipitated by adding isopropanol and then dissolved in water. To promote the correct folding of the aptamer, the RNA solution was incubated at $75^{\circ} \mathrm{C}$ for $2 \mathrm{~min}$, and slowly cooled to $25^{\circ} \mathrm{C}$ over $10 \mathrm{~min}$. Then, $1 / 5$ volume of $6 \times$ aptamer selection buffer (ASB) containing $120 \mathrm{mM}$ HEPES (pH 7.4), 30 $\mathrm{mM} \mathrm{MgCl} 2$ and $750 \mathrm{mM} \mathrm{KCl}$ was added and the RNA library was incubated at $25^{\circ} \mathrm{C}$ for another $10 \mathrm{~min}$.

TMR-decorated beads were prepared by mixing TMR-SS-Biotin (dissolved in water) with streptavidin-beads $(0.2 \mathrm{~mL})$ in $1 \times$ ASB. After $5 \mathrm{~min}$, TMR-beads were washed with $1 \times$ ASB to remove excess, unbound TMR-SS-Biotin (if any). Next, the folded RNA was mixed with the TMR-beads and shaken for $1 \mathrm{~h}$ at $25^{\circ} \mathrm{C}$. To remove unbound and low affinity aptamers, the resin was washed with seven volumes of $1 \times$ ASB and the TMR-bound RNA was eluted with $20 \mathrm{mM}$ DTT which cleaves the disulfide bond between TMR and biotin. The eluted RNA was ethanol precipitated with glycogen $(20 \mu \mathrm{g})$, reversetranscribed using SuperScript III (Invitrogen), PCR amplified for 10 cycles and the purified double-stranded 
DNA template was subjected to the next round for selection. Seven rounds of SELEX were performed using the described protocol with progressively increased stringency. The TMR concentration on the streptavidin resin was decreased from $40 \mu \mathrm{M}$ (round 1) to $10 \mu \mathrm{M}$ (rounds $2-7$ ) and the RNA concentration was decreased from $20 \mu \mathrm{M}$ (round 1) to $10 \mu \mathrm{M}$ (rounds $2-4$ ) and $5 \mu \mathrm{M}$ (rounds $5-7$ ), whereas the number of washes to remove low affinity binders from the resin was increased to 14 volumes of $1 \times$ ASB in the final round. After each round of SELEX, the efficiency of the selection was monitored by quantifying the amount of eluted RNA and measuring the fluorescence enhancements of TMR-DN (25 nM) upon addition of the RNA pool (500 nM). After seven rounds, the double stranded DNA was cloned into a vector, which was subsequently transformed into bacteria, and 96 individual colonies were sent for Sanger sequencing.

Screening of SRB-2 mutants for fluorescence enhancement. Before fluorescence measurements, SRB-2 mutants $\left(1.2 \mu \mathrm{M}\right.$, dissolved in water) were incubated at $75^{\circ} \mathrm{C}$ for $2 \mathrm{~min}$, and then cooled to $25^{\circ} \mathrm{C}$ within 10 min to promote the correct folding of the aptamer. Then, $1 / 5$ volume of $6 \times$ ASB was added and the aptamer solution was incubated at $25^{\circ} \mathrm{C}$ for an additional $10 \mathrm{~min}$. The fluorescence intensity of the aptamer solutions after addition of TMR-DN (20 nM) was recorded, and divided by the fluorescence of TMR-DN without any aptamer to yield fluorescence enhancement values of each mutant. The best mutants were further characterized in detail.

In vitro characterization of SRB aptamers. Aptamers were always folded as described above and all properties were measured in $20 \mathrm{mM} \mathrm{HEPES} \mathrm{(pH7.4),} 125 \mathrm{mM} \mathrm{KCl}$ and $1 \mathrm{mM} \mathrm{MgCl}$ buffer unless specified. For fluorescence measurements, the excitation and emission maxima of the aptamer:TMR-DN complexes were used. The fluorescence turn-on value is defined as the ratio of the emission peak intensity of the fluorophore $(20 \mathrm{nM})$ in the presence of SRB aptamers $(500 \mathrm{nM})$ to that of the fluorophore $(20 \mathrm{nM})$ in the absence of SRB aptamers.

Dissociation constants $\left(K_{\mathrm{d}}\right)$ for aptamer:TMR-DN complexes were determined by measuring the increase in the fluorescence intensity as a function of increasing RNA concentration in the presence of a 
fixed amount of TMR-DN (1 nM) at $25^{\circ} \mathrm{C}$. Dissociation constants were calculated after fitting the curves to equation 1 by using least-squared fitting (OriginPro 8.5.1):

$$
F=F_{0}+\frac{\left(F_{\infty}-F_{0}\right) \times\left\{\left(K_{D}+P_{0}+[A p t]\right)-\sqrt{\left([A p t]-P_{0}\right)^{2}+K_{D} \times\left(K_{D}+2[A p t]+2 P_{0}\right)}\right\}}{2 P_{0}}
$$

where $F$ is the fluorescence at any given aptamer concentration, $F_{0}$ is the fluorescence of the free TMRDN with initial concentration of $P_{0}, F_{\infty}$ is the maximum fluorescence intensity reached when all TMR-DN is in complex with the aptamer, $[A p t]$ is the final concentration of added aptamer and $K_{D}$ is the equilibrium dissociation constant.

To determine the $\mathrm{Mg}^{2+}$ dependence, $1 / 5$ volume of $6 \times$ ASB buffer containing different amounts of $\mathrm{MgCl}_{2}(0-10 \mathrm{mM})$ was added to the RNA (600 nM in water, already incubated at $75{ }^{\circ} \mathrm{C}$ for 2 min and cooled to $25^{\circ} \mathrm{C}$ ) and incubated for $10 \mathrm{~min}$ at $25^{\circ} \mathrm{C}$.

To determine the dependence of the aptamer:TMR-DN fluorescence on monovalent cations $\left(\mathrm{Li}^{+}\right.$, $\left.\mathrm{Na}^{+}, \mathrm{K}^{+}\right), 1 / 5$ volume of a $6 \times$ buffer containing $750 \mathrm{mM}$ of the corresponding cation as chloride salt or without any monovalent cation, $120 \mathrm{mM}$ HEPES ( $\mathrm{pH}$ 7.4) and $6 \mathrm{mM} \mathrm{MgCl}_{2}$ was added to the aptamer solutions (600 nM in water, already incubated at $75^{\circ} \mathrm{C}$ for 2 min and cooled to $25^{\circ} \mathrm{C}$ ) and incubated for $10 \mathrm{~min}$ at $25^{\circ} \mathrm{C}$. Fluorescence intensities were measured upon addition of TMR-DN (500 nM).

To study the temperature dependence of the aptamer:TMR-DN complexes, folded aptamers (500 $\mathrm{nM})$ were incubated with TMR-DN (500 nM) for $10 \mathrm{~min}$. Then, the fluorescence decay was recorded upon increasing the temperature from $25^{\circ} \mathrm{C}$ to $65^{\circ} \mathrm{C}\left(0.5^{\circ} \mathrm{C} / \mathrm{min}\right)$.

To obtain CD spectra, aptamers $(5 \mu \mathrm{M})$ were folded in $20 \mathrm{mM} \mathrm{Na} \mathrm{HPO}_{4} / \mathrm{NaH}_{2} \mathrm{PO}_{4}(\mathrm{pH} 7.2), 125$ $\mathrm{mM} \mathrm{KCl}$ and $1 \mathrm{mM} \mathrm{MgCl}_{2}$ as previously described and incubated at $25^{\circ} \mathrm{C}$ for $10 \mathrm{~min}$. Measurements were performed at $25^{\circ} \mathrm{C}$ with a $1 \mathrm{~nm}$ resolution using a $1-\mathrm{mm}$ cell. CD melting curves of the aptamers $(5 \mu \mathrm{M})$ were recorded upon increasing the temperature from $25^{\circ} \mathrm{C}$ to $98^{\circ} \mathrm{C}\left(2^{\circ} \mathrm{C} / \mathrm{min}\right)$ by monitoring the amplitude at $268 \mathrm{~nm}$.

The folding assay was carried out using a slightly modified procedure of Strack et al ${ }^{21}$. Briefly, the fluorescence intensity of a mixture containing $500 \mathrm{nM}$ aptamer (10-fold in excess) and $50 \mathrm{nM}$ TMR-DN was measured $\left(F_{1}\right)$ where the amount of formed complex was controlled by the limiting component TMRDN. Next, the fluorescence intensity of a mixture containing $50 \mathrm{nM}$ aptamer and $500 \mathrm{nM}$ TMR-DN (10-fold 
in excess) was measured $\left(F_{2}\right)$, where the amount of formed complex is controlled by the limiting component SRB. Finally, the fluorescence intensity of $500 \mathrm{nM}$ TMR-DN was measured $\left(F_{3}\right)$ for fluorescence background corrections due to unbound excess TMR-DN. Fluorescence intensities were measured in 20 mM HEPES ( $\mathrm{pH} 7.4), 1 \mathrm{mM} \mathrm{MgCl} 2$ and $125 \mathrm{mM} \mathrm{KCl}$ at $25^{\circ} \mathrm{C}$. The fraction of the correctly folded aptamer ( $f$ ) was calculated by using equation 2 :

$$
f=\frac{F_{2}-F_{3}}{F_{1}-0.1 \times F_{3}}
$$

Quantum yields (QYs) of the aptamer:TMR-DN complexes were determined using a crosscalibrated sulforhodamine 101 standard $(Q Y=1.00$ in ethanol). The integral of the fluorescence emission spectra and the absorbance of the samples at the excitation wavelength ( $528 \mathrm{~nm}$ ) were measured for at least four different concentrations of the compounds. To avoid inner filter effects in the fluorescence measurements, concentrations with an absorbance below 0.05 were used. The obtained values were plotted in a graph (fluorescence integral vs. absorbance). The slope of the linear fit was compared with that of the reference sample and the QYs were determined using equation 3:

$$
Q Y=Q Y_{R}\left(\frac{m}{m_{R}}\right)\left(\frac{n^{2}}{n_{R}^{2}}\right)
$$

where $m$ is the slope, $n$ is the refractive index of the solvent and the subscript $R$ refers to the reference fluorophore with a known quantum yield.

Photobleaching experiments were performed using a LED setup equipped with a Luxeon Rebel ES Lime LED (nominal wavelength $=567 \mathrm{~nm}, \mathrm{U}=3.0 \mathrm{~V}, \mathrm{I}=800 \mathrm{~mA}$ ). JF $549-$ Halo fluorophore ${ }^{22}$ and $50 \%$ of the mRuby $3^{23}$ fluorescent protein were prepared in $20 \mathrm{mM} \mathrm{HEPES}(\mathrm{pH} 7.4), 1 \mathrm{mM} \mathrm{MgCl} 2,125 \mathrm{mM} \mathrm{KCl}$, and $0.05 \%$ Tween-20 at a final concentration of $20 \mathrm{nM}$. RhoBAST:TMR-DN was freshly prepared by mixing $500 \mathrm{nM}$ of folded RhoBAST and $20 \mathrm{nM}$ of TMR-DN. All samples were continuously irradiated and the corresponding fluorescence was measured every 20 min over a period of $3 \mathrm{~h}$.

DNA cloning. For bacterial expression, double-stranded DNA carrying SRB-2, SRB-3 and RhoBAST sequences (Supplementary Table 3) flanked by EcoRI and Sall restriction enzyme sites were created in a PCR reaction, double digested and ligated into EcoRI/Sall double digested pET-GFP plasmid (Addgene, 
Plasmid \# 29663) to form pET-GFP-SRB-2, pET-GFP-SRB-3 and pET-GFP-RhoBAST plasmids. The chloramphenycol acetyl transferase (cat) gene was PCR amplified from the pLysS plasmid (Thermo Fisher Scientific, isolated from BL21(DE3)pLysS E. coli) and cloned into the HindlII and EcoRI sites of pTACMAT-Tag2 (Sigma-Aldrich) to yield the pTac-Cat plasmid. A single-stranded, ultramer oligonucleotide containing two repeats of synonymous RhoBAST (Supplementary Table 3) flanked by Sall and Xhol sites was synthesized, PCR amplified and blunt-end ligated into PCR-linearized pTac-Cat to yield pTac-CatRhoBAST 2 . Another ultramer containing two repeats of synonymous RhoBAST flanked by Sall and Xhol sites was synthesized, PCR amplified, double digested with Sall and Xhol and cloned into Xhol-digested and dephosphorylated pTac-Cat-RhoBAST 2 to yield pTac-Cat-RhoBAST 4 . A cassette containing four repeats of RhoBAST can be obtained by PCR using pTac-Cat-RhoBAST 4 as a template. RhoBAST 4 was then double-digested with Sall and Xhol, and cloned into Xhol-digested and dephosprylated pTac-CatRhoBAST $_{4}$ to yield pTac-Cat-RhoBAST 8 . A cassette containing eight repeats of RhoBAST can be obtained by Sall and Xhol double digestion of pTac-Cat-RhoBAST 8 followed by gel-purification. Cloning this cassette into Xhol-digested and dephosprylated pTac-Cat-RhoBAST 8 yielded pTac-Cat-RhoBAST ${ }_{16}$.

The GFP gene carrying HindIII at $5^{\prime}$ and Sall-Sphl at $3^{\prime}$ was PCR amplified from the pET-GFP plasmid and cloned into HindlII and Sphl sites of pTAC-MAT-Tag2 to yield pTac-GFP. A cassette containing 16 repeats of RhoBAST obtained by double digestion and gel purification of pTac-CatRhoBAST $_{16}$. It was then cloned into Sall digested and dephosphorylated pTac-GFP to yield pTac-GFPRhoBAST $_{16}$. TolB (MKQALRVAFGFLILWASVLHA), DsbA (MKKIWLALAGLVLAFSASA) and PhoA (MKQSTIALALLPLLFTPVTKA) signal peptide sequences were fused to the N-terminus of GFP in pTacGFP-RhoBAST ${ }_{16}$ by PCR. Linearized-plasmids were then phosphorylated and self-ligated to form pTacTolB-GFP-RhoBAST 16 , pTac-DsbA-GFP-RhoBAST ${ }_{16}$, and pTac-PhoA-GFP-RhoBAST 16.

For mammalian expression, an ultramer ssDNA containing two repeats of synonymous RhoBAST was synthesized, PCR amplified and cloned between Sall and Xbal sites of pAV-U6+27 (Addgene, plasmid \#25709) to yield pAV-U6+27-RhoBAST 2 . To generate pAV-Tornado-RhoBAST, an ultramer ssDNA containing RhoBAST sequence embedded into the Tornado system ${ }^{14}$ was synthesized, PCR amplified and cloned between the Sall and Xbal sites of pAV-U6+27. pUC19-CAG-GFP was prepared by digesting the GFP gene from the AAVS1-mEGFP (Addgene, plasmid \#91565) plasmid by using Sdal and Notl and 
cloning it into PCR-linearized pUC19. A cassette containing RhoBAST ${ }_{16}$ was obtained by Sall and Xhol digestion and gel purification of pTac-Cat-RhoBAST ${ }_{16}$. It was then blunted and cloned into pUC19-CAGGFP which was already digested with the Mlul enzyme, blunted and dephosphorylated to yield pUC19CAG-GFP-RhoBAST ${ }_{16}$. Blunt-end repaired RhoBAST ${ }_{16}$ was cloned into 5' UTR CGG 99x FMR1-EGFP (Addgene, plasmid \#63091) which was digested with the Notl enzyme, blunted and dephosphorylated, to yield 5' UTR CGG 99x FMR1-EGFP-RhoBAST 16.

E. coli K12 genome editing. To genomically label ompA and rnaseE with RhoBAST arrays at the 3' UTR, double stranded DNA cassettes containing a left-homologous arm, RhoBAST $_{16}$, a terminator, a kanamycin expression system, and a right-homologous arm sequences were prepared by PCR and ligation reactions (Supplementary Table 4). On the other hand, the double-stranded DNA cassette for the insertion of cat gene into lacz locus was prepared by the double digestion of pTac-Cat-RhoBAST 16 plasmid with BamHI and Pagl, gel-purification of the cat-RhoBAST ${ }_{16}$ expression system, which was subsequently ligated to a left-homologous arm carrying a synthetic terminator at the 5'-end and to a right homologous arm at the 3'end (Supplementary Table 4). Red/ET homologous recombination in E. coli K12 was established using the double stranded DNA casettes prepared as described above and the Quick and Easy E. coli Gene Deletion Kit (Gene Bridges) according to manufacturers' instructions. Selection of bacteria with modified genomes was accomplished in the presence of kanamycin for ompA and rnase $E$ or chloramphenicol for cat. Successful modifications of the genome were verified by PCR.

Preparation of 5'-Biotin and 3'-Cy5 labeled RhoBAST. T7 RNA polymerase was added into a transcription mixture containing the double-stranded DNA template (5'TCTAATACGACTCACTATTAGGAACCTCCGCGAAAGCGGTGAAGGAGAGGCGCAAGGTTAACCGC CTCAGGTTCCAA-3', the sequence of T7 $\varphi 2.5$ promoter is underlined, $1 \mu \mathrm{M})$, ATP $(0.5 \mathrm{mM})$, BiotinHDAAMP (2 mM), CTP (1 mM), GTP (1 mM), UTP (1 mM), DTT (10 mM), spermidine (2 mM), Tris- $\mathrm{HCl}$ (40 mM, pH 8.1), $\mathrm{MgCl}_{2}(22 \mathrm{mM})$, Triton-X-100 (0.01\%), BSA (40 $\left.\mu \mathrm{g} / \mathrm{mL}\right)$ and pyrophosphatase $(1 \mathrm{U} / \mathrm{mL})$. After $4 \mathrm{~h}$ at $37^{\circ} \mathrm{C}$, the reaction mixture was treated with DNasel $(50 \mathrm{U} / \mathrm{mL})$ for $30 \mathrm{~min}$ at $37^{\circ} \mathrm{C}$. RNA was purified by electrophoresis on a $10 \%$ denaturing polyacrylamide gel, excised from the gel, ethanol 
precipitated and dissolved in water. The percentage of biotinylation ( $30 \%)$ was determined by streptavidin-shift assay. 3' of Biotin-RhoBAST was further ligated to Cy5, by incubating the RNA (10 $\mu \mathrm{M})$ with ATP $(1 \mathrm{mM})$, pCp-Cy5 $(20 \mu \mathrm{M})$ and T4 RNA ligase at $4{ }^{\circ} \mathrm{C}$ overnight. RNA was then extracted with phenol:chloroform:isoamyl alcohol (25:24:1, pH 4.5), precipitated using sodium acetate and ethanol, dissolved in water and stored at $-20^{\circ} \mathrm{C}$. The percentage of Cy5 labeling ( 60\%) was calculated by measuring the absorbtion of the Cy5 labeled RNA at $648 \mathrm{~nm}$ (extinction coefficient of Cy5 at $648 \mathrm{~nm}$ is $\left.250,000 \mathrm{M}^{-1} \mathrm{~cm}^{-1}\right)$.

Live-cell confocal imaging of bacterial cells. BL21 Star ${ }^{\mathrm{TM}}$ (DE3) competent E. coli cells were transformed with either pET-GFP (control plasmid) or pET-GFP-SRB-2, -3 or pET-GFP-RhoBAST plasmids. Next day, single colonies were picked from LB-agar/Kanamycin $(30 \mu \mathrm{g} / \mathrm{mL})$ plates and grown in $5 \mathrm{~mL}$ of LB medium containing kanamycin $(30 \mu \mathrm{g} / \mathrm{mL})$ overnight at $37^{\circ} \mathrm{C}$ with shaking at $150 \mathrm{rpm}$. A fresh culture with an $\mathrm{OD}_{600}$ of 0.01 was started using the overnight culture as a starter culture in a $50 \mathrm{~mL}$ falcon flask containing $10 \mathrm{ml}$ of LB medium with $30 \mu \mathrm{g} / \mu \mathrm{L}$ kanamycin. When $\mathrm{OD}_{600}$ was 0.4 , IPTG (at desired concentration, $\leq 1 \mathrm{mM}$ ) was added into the culture, and flasks were shaken for an additional $2-3 \mathrm{~h}$ at 37 ${ }^{\circ} \mathrm{C}$. Then, $200 \mu \mathrm{L}$ of the culture was removed, spun down and resuspended in $1 \mathrm{~mL}$ of M9 medium. 200 $\mu \mathrm{L}$ of this suspension was transferred into a poly-D-lysine coated 8-well glass chamber and incubated at $37^{\circ} \mathrm{C}$ for $20 \mathrm{~min}$. Finally, the wells were gently washed twice and bacteria were incubated with $100 \mathrm{nM}$ of TMR-DN in M9 medium at $37^{\circ} \mathrm{C}$. Bacteria were imaged after 20 min of incubation at $37^{\circ} \mathrm{C}$.

Images were taken on a point-scanning confocal microscope with hybrid scanner (galvo/resonant) equipped with a Nikon N Apo 60x NA $1.4 \lambda$ s OI (WD $0.14 \mathrm{~mm}$, FOV 0.21 x $0.21 \mathrm{~mm}^{2}$ ) objective. A $405 \mathrm{~nm}$ laser was used to excite Hoechst 33342 (detection via an emission filter set of $450 \pm 25 \mathrm{~nm}$ ), a $488 \mathrm{~nm}$ laser was used to excite GFP (emission filter set $525 \pm 25 \mathrm{~nm}$ ) and a $561 \mathrm{~nm}$ laser was used to excite TMR (emission filter set $595 \pm 25 \mathrm{~nm}$ ). The laser settings were optimized for each condition. Z-stack images were taken with a step size of $250 \mathrm{~nm}$. Images were analyzed by Fiji/lmageJ and the background correction was done by subtracting the mean fluorescence intensity of a surface area without attached $E$. coli cells from the whole image. The mean fluorescence intensity was determined from defined areas at the poles of the bacteria. 
For imaging GFP mRNA using pTac-GFP (control plasmid), pTac-GFP-RhoBAST ${ }_{16}$, pTac-TolBGFP-RhoBAST $_{16}$, pTac-DsbA-GFP-RhoBAST $_{16}$ and pTac-PhoA-GFP-RhoBAST 16 plasmids, DH5a cells were used and the LB growth medium was supplemented with ampicillin $(100 \mu \mathrm{g} / \mathrm{mL})$ instead of kanamycin.

For imaging ompA and rnaseE mRNA in E. coli K12, LB growth medium was supplemented with kanamycin $(15 \mu \mathrm{g} / \mathrm{mL})$. For imaging cat mRNA in E. coli K12, LB growth medium was supplemented with chloramphenicol $(10 \mu \mathrm{g} / \mathrm{mL})$.

Confocal imaging of fixed bacterial cells. $700 \mu \mathrm{L}$ bacteria expressing RNA of interest fused to RhoBAST in LB or M9 medium (as described above) were quickly mixed with $100 \mu \mathrm{L} 8 \%$ paraformaldehyde (PFA) solution, and incubated for $10 \mathrm{~min}$ at room temperature. Bacteria were then pelleted by centrifugation, washed twice with $1 \mathrm{~mL}$ MgPBS (DPBS containing $1 \mathrm{mM} \mathrm{MgCl}_{2}$ ), resuspended in $1 \mathrm{~mL} \mathrm{MgPBS}$ and stored overnight at $4{ }^{\circ} \mathrm{C}$. On the next day, $200 \mu \mathrm{L}$ of the culture was transferred into a poly-D-lysine coated 8-well glass chamber and incubated at room temperature for $20 \mathrm{~min}$. Finally, the wells were gently washed twice and bacteria were incubated with $1 \mu \mathrm{g} / \mathrm{mL}$ Hoechst 33342 and $50 \mathrm{nM}$ TMR-DN in MgPBS (or M9 medium) at $37^{\circ} \mathrm{C}$ for $20 \mathrm{~min}$. Bacteria were then imaged at room temperature.

Quantitative PCR with reverse transcription (RT-qPCR). Chemically competent DH5a competent $E$. coli cells (Thermo Fisher Scientific) were transformed with pTac-GFP (negative control) and pTac-GFPRhoBAST $_{16}$. On the next day, single colonies were picked from the LB-agar/ampicillin $(100 \mu \mathrm{g} / \mathrm{mL})$ plates and grown overnight in $5 \mathrm{~mL}$ of LB medium containing ampicillin $(100 \mu \mathrm{g} / \mathrm{mL})$ at $37^{\circ} \mathrm{C}$ with shaking. A fresh culture with an $\mathrm{OD}_{600}$ of 0.01 was started using the overnight culture as a starter in a $50 \mathrm{~mL}$ conical falcon flask containing $15 \mathrm{~mL}$ of LB medium with $100 \mu \mathrm{g} / \mu \mathrm{L}$ ampicillin. After $3 \mathrm{~h}\left(\mathrm{OD}_{600} \sim 0.4\right)$, the culture was equally divided into 3 different falcon flasks and different amounts of IPTG $(0,10 \mu \mathrm{M}, 40 \mu \mathrm{M})$ were added into each culture flask. Cells were shaken for another $2 \mathrm{~h}$ at $37^{\circ} \mathrm{C}$. Then, $2 \mathrm{~mL}$ of the cultures were spun down, LB medium was carefully removed from the bacteria pellet and the pellet was resuspended in 450 $\mu \mathrm{L}$ of $\mathrm{M9}$ medium. $400 \mu \mathrm{L}$ of this mixture were used for total RNA isolation and $50 \mu \mathrm{L}$ were used for fixed- 
cell imaging. Total RNA from the bacteria was isolated using RNAzol ${ }^{\circledR}$ RT according to the manufacturers' instructions and obtained RNA pellet was finally dissolved in $200 \mu \mathrm{L}$ of water.

Total RNA $(20 \mu \mathrm{L}, \sim 2.6 \mu \mathrm{g})$ was then subjected to DNasel $(50 \mathrm{U} / \mathrm{mL})$ treatment in $60 \mu \mathrm{L}$ total volume at $37^{\circ} \mathrm{C}$ for $1 \mathrm{~h}$ and the DNasel was inactivated by adding $4 \mu \mathrm{L}$ of $50 \mathrm{mM}$ EDTA and incubating at $65^{\circ} \mathrm{C}$ for 10 min. CDNA was produced using $5 \mu \mathrm{L}$ of each RNA sample and SuperScript ${ }^{\mathrm{TM}}$ IV-RT (Invitrogen) according to manufacturers' instructions. Reverse-transcription reactions were diluted 10-fold with water prior to qPCR which was performed in a Light Cycler 480 instrument (Roche) using the Brilliant III Ultra-Fast SYBR® Green qPCR Mastermix (Agilent). Forward and reverse GFP primers are 5'TGCTGCTGCCCGACAACCAC-3' and 5'-CGGTCACGAACTCCAGCAGGAC-3', respectively. qPCR data were analyzed with the LightCycler 480 software. As negative controls, no-RT and no-template reactions were used. All reactions were performed in triplicate on the $20 \mu \mathrm{L}$ scale. $16 \mathrm{~S}$ rRNA was used as an internal reference gene. For absolute quantification of RNA copy numbers, a small fragment ( 180 nucleotide) of GFP gene was in vitro transcribed and used as a reference RNA in RT-qPCR.

Confocal imaging of live mammalian cells. HeLa or HEK293T cells were cultured at $37{ }^{\circ} \mathrm{C}$ under $5 \%$ $\mathrm{CO}_{2}$ in Dulbecco's Modified Eagle's Medium (with $4.5 \mathrm{~g} / \mathrm{L}$ high glucose, $4 \mathrm{mM}$ L-glutamine, $25 \mathrm{mM}$ HEPES and without phenol red) supplemented with $10 \%$ FBS, $100 \mathrm{unit} / \mathrm{mL}$ penicillin and $100 \mu \mathrm{g} / \mathrm{mL}$ streptomycin. To improve the adherence of HEK293T cells, glass slides were coated with poly-D-lysine before plating the cells.

For imaging experiments, $3 \times 10^{4}$ cells were seeded overnight in $\mu$-Slide chambered coverslips with 8-wells containing $300 \mu \mathrm{L}$ of medium each. The following day, cells were transfected with the appropriate plasmid using the FuGeneHD transfection reagent (Promega) according to the manufacturer's protocol. After $48 \mathrm{~h}$ at $37^{\circ} \mathrm{C}$, the medium was exchanged with Leibowitz (L15) medium containing $1 \mu \mathrm{gg} / \mathrm{mL}$ Hoechst 33342 and $100 \mathrm{nM}$ of TMR-DN. After incubation for $1 \mathrm{~h}$, cells were imaged at $37^{\circ} \mathrm{C}$ in a live-cell imaging chamber with controlled humidity.

Images were taken as described for bacterial cells above, except for the following modifications. Z-stack images were taken with a step size of $500 \mathrm{~nm}$. Images were analyzed by Fiji/lmageJ and the 
background correction was done by subtracting the mean fluorescence intensity of a surface area without adherent cells from the whole image.

SIM imaging of bacterial cells. DH5a cells E. coli cells were transformed with either pTac-GFP (control plasmid) or pTac-GFP-RhoBAST 16 , pTac-TolB-GFP-RhoBAST $_{16}$ or pTac-PhoA-GFP-RhoBAST $_{16}$ plasmids. On the following day, single colonies were picked from LB-agar/Ampicillin (100 $\mu \mathrm{g} / \mathrm{mL})$ plates and grown in $5 \mathrm{~mL}$ of LB medium containing ampicillin $(100 \mu \mathrm{g} / \mathrm{mL})$ overnight at $37^{\circ} \mathrm{C}$ with shaking at $150 \mathrm{rpm}$. A fresh culture with an $\mathrm{OD}_{600}$ of 0.01 was started using the overnight culture as a starter culture in a $50 \mathrm{~mL}$ falcon flask containing $10 \mathrm{~mL}$ of LB medium with $100 \mu \mathrm{g} / \mu \mathrm{L}$ ampicillin. When $\mathrm{OD}_{600}$ was 0.4 , IPTG $(40 \mu \mathrm{M})$ was added into the culture, and flasks were shaken for an additional $2-3 \mathrm{~h}$ at $37^{\circ} \mathrm{C}$. Then, $200 \mu \mathrm{L}$ of the culture was removed, spun down and resuspended in $1 \mathrm{~mL}$ of $\mathrm{M} 9$ medium. At this point cells can be fixed using PFA (as described before) and imaged later. For live-cell imaging, $200 \mu \mathrm{L}$ of this suspension was transferred into a poly-D-lysine coated 8-well glass chamber and incubated at $37^{\circ} \mathrm{C}$ for 20 min. Finally, the wells were gently washed twice and bacteria were incubated with $50 \mathrm{nM}$ of TMR-DN in M9 medium at $37^{\circ} \mathrm{C}$. Bacteria were imaged after $20 \mathrm{~min}$ of incubation at $37^{\circ} \mathrm{C}$.

Images were taken on a Nikon N-SIM system equipped with total internal reflection fluorescence Apochromat $100 \times 1.49$ NA oil immersion objective and an electron-multiplying charge-coupled device camera with single-molecule sensitivity (iXon3 DU897E; Andor Technology). A $488 \mathrm{~nm}$ laser was used to excite GFP (detection via an emission filter set of 520/45 nm), a $561 \mathrm{~nm}$ laser was used to excite TMR (emission filter set 610/60 nm). The laser settings were optimized for each condition. Images were taken sequentially within a small z-stack (step size of $150 \mathrm{~nm}$ ). Subsequently, reconstruction of the superresolution images was performed with the NIS imaging and image analysis software (Nikon).

Imaging of immunostained mammalian cells. $3 \times 10^{4}$ HEK293T cells were seeded overnight in poly-Dlysine-coated $\mu$-Slide (chambered coverslip) with 8 wells containing $300 \mu \mathrm{L}$ of medium. On the following day, cells were transfected with pAV-Tornado-RhoBAST plasmid ( 200 ng/well) using FuGeneHD transfection reagent (Promega) according to the manufacturer's protocol. After $48 \mathrm{~h}$ at $37^{\circ} \mathrm{C}$, the medium was exchanged with $300 \mu \mathrm{L}$ L15 medium. Then, half of the medium was removed, $150 \mu \mathrm{L} 8 \%$ PFA (in 
DPBS, pH 7.4) was added and the solution was gently but quickly mixed. After 10 min at room temperature, cells were washed 3 times with $500 \mu \mathrm{L}$ of MgPBS. In order to permeabilize the cells, $400 \mu \mathrm{L}$ of $0.1 \%$ Triton $\mathrm{X}-100$ in MgPBS was added into the wells and cells were incubated for $15 \mathrm{~min}$ at room temperature. TritonX-100 was removed and the cells were washed 3 times with $500 \mu \mathrm{L}$ MgPBS. Before proceeding to immunostaining, cells were incubated with $500 \mu \mathrm{L}$ of $2 \%$ BSA in MgPBS overnight at $4{ }^{\circ} \mathrm{C}$ for blocking. On the next day, the appropriate concentration of primary antibody diluted in $500 \mu \mathrm{L}$ of $0.1 \%$ BSA was added to the cells and incubated for $1-2 \mathrm{~h}$ at room temperature. All antibodies used in this study and their dilution factors are listed in Supplementary Table 5. The primary antibody solution was removed from the wells and the cells were washed three times with $500 \mu \mathrm{L}$ of MgPBS for $5 \mathrm{~min}$. Then, the appropriate concentration of the Alexa488 fluorophore-labeled secondary antibody diluted in $500 \mu \mathrm{L}$ of $0.1 \%$ BSA was added to the cells and incubated for $1 \mathrm{~h}$ at room temperature. Finally, the secondary antibody solution was removed from the wells and the cells were washed three times with $500 \mu \mathrm{L}$ of MgPBS for 5 min. Before imaging, the solution was exchanged with MgPBS containing $1 \mu \mathrm{g} / \mathrm{mL}$ Hoechst 33342 and $100 \mathrm{nM}$ of TMRDN and the cells were incubated for $10 \mathrm{~min}$ at room temperature.

Images were taken as described before for live mammalian cells; imaging conditions identical to those of GFP were used for Alexa488.

Single-molecule studies of RhoBAST binding kinetics. A home-built total internal reflection fluorescence (TIRF) microscope based on an inverted microscope (Axiovert 200, Zeiss, Göttingen, Germany) ${ }^{24}$ was used. Light from a $638 \mathrm{~nm}$ laser (MLD 638, Cobolt AB, Solna, Schweden) and a $561 \mathrm{~nm}$ laser (Jive, Cobolt AB) is combined via dichroic mirrors into an acousto-optical tunable filter (AOTFnC400.650, A-A Opto-Electronic, Orsay, France) for precise and fast control of the laser intensities. The light is guided into a Pellin-Broca prism located on top of a quartz glass sample slide and totally reflected at the boundary between the sample slide and the aqueous sample solution. To ensure undisturbed guidance of the beams to the boundary layer, immersion oil ( $n=1.518$, Immersol $518 \mathrm{~F}$, Zeiss) is used between the prism and the quartz glass sample slide. Fluorescence emission is collected through a water immersion objective (C-Apochromat 63x/1.2 W Corr M27, Zeiss). Excitation light in the detection path is blocked by 
561 and $635 \mathrm{~nm}$ notch filters (Thorlabs, Newton, NJ) and the fluorescence emission is detected by an EMCCD camera (Ixon EM+ DU-897, Andor, Belfast, UK) with a pixel size of $120 \times 120 \mathrm{~nm}^{2}$.

In order to prepare the sample slides, $75 \times 75 \times 1 \mathrm{~mm}^{3}$ quartz slides (Finkenbeiner, Scientific Glass Blower, Waltham, MA) were sonicated for $30 \mathrm{~min}$ at $60^{\circ} \mathrm{C}$ in $1 \mathrm{M} \mathrm{KOH}$. This step was repeated with fresh $1 \mathrm{M} \mathrm{KOH}$ solution. Afterwards, they were sonicated for $15 \mathrm{~min}$ at $60{ }^{\circ} \mathrm{C}$ in Millipore water. This step was repeated after water exchange. Then, the slides were dried under nitrogen and plasma-cleaned for $30 \mathrm{~min}$, and incubated overnight in $0.05 \%(\mathrm{v} / \mathrm{v})$ dichlorodimethylsilane (Sigma-Aldrich, St. Louis, MO) solution in n-hexane (VWR, Radnor, PA). On the following day, the slides were sonicated in $n$-hexane at room temperature for 1 min three times in succession, each time with fresh $n$-hexane.

Cover slips $\left(20 \times 20 \times 0.17 \mathrm{~mm}^{3}\right.$, Menzel Gläser, Thermo Fischer, Waltham, MA) were prepared in essentially the same way, except that plasma cleaning was omitted. Quartz glass slides and cover slips were stored at $4{ }^{\circ} \mathrm{C}$ until use.

The quartz slides and cover slips were glued together using two pieces of double-sided adhesive tape, leaving a $3 \mathrm{~mm}$ wide channel in the middle. The channel was filled with $20 \mu \mathrm{L}$ of a $0.01 \mathrm{mg} / \mathrm{mL}$ neutravidin solution in PBS and incubated for $10 \mathrm{~min}$. Then, the channel was washed with $100 \mu \mathrm{L}$ PBS and incubated for $10 \mathrm{~min}$ with $100 \mu \mathrm{L}$ of a $0.1 \mathrm{mg} / \mathrm{mL}$ solution of Lutensol AT50 (BASF SE, Ludwigshafen, Germany) in water for surface passivation. The channel was washed again with $100 \mu \mathrm{L}$ of PBS. To ensure a proper folding of the aptamer, the RhoBAST stock solution (20 nM in water) was heated to $75{ }^{\circ} \mathrm{C}$ for 2 min and then slowly cooled to room temperature prior to filling it into the channel. To obtain a sparse decoration of the surface with aptamers, the Cy5-labelled, biotinylated RhoBAST stock was diluted to a final concentration of $25 \mathrm{pM}$ with $1 \times \mathrm{ASB}$. The channel was flushed with $100 \mu \mathrm{L}$ of this solution and incubated for $10 \mathrm{~min}$. Then, the channel was washed with $100 \mu \mathrm{L}$ ASB and filled with $20 \mu \mathrm{L}$ of imaging buffer containing the TMR-DN dye at the desired concentration, an enzymatic oxygen scavenging system (1.4 mg/mL glucose oxidase, $0.03 \mathrm{mg} / \mathrm{mL}$ catalase, $10 \%(\mathrm{w} / \mathrm{w})$ glucose), $1 \mathrm{mM}$ Trolox as triplet state quencher and $0.1 \%(\mathrm{v} / \mathrm{v})$ Tween 20 in $1 \times \mathrm{ASB}$. The channel was sealed with transparent nail polish after adding the imaging solution and measurements were started after $10 \mathrm{~min}$ to ensure equilibration.

To locate RhoBAST molecules, 100 camera frames (100 ms dwell time) were recorded with 638 $\mathrm{nm}$ excitation ( $7 \mathrm{~mW}$ in front of the prism) to excite the Cy5 marker attached to the RhoBAST molecules. 
Then, the Cy5 was photobleached using $49 \mathrm{~mW}$ laser power to avoid energy transfer from bound TMRDN dyes to the Cy5 label in the subsequent measurement. Afterwards, the TMR-DN dyes were imaged with $561 \mathrm{~nm}$ excitation, taking 18,000 frames (100 ms dwell time) with a laser power between 2 and $7 \mathrm{~mW}$. Images and time traces were analyzed in a semi-automatic way using our own software written in MATLAB 2019a (MathWorks, Natick, MA). Aptamer locations were identified as local intensity maxima in the images taken in the Cy5 channel. Only maxima with an intensity of $80 \%$ or more of the global maximum were included. The intensities in the TMR-DN channel were integrated within a $5 \times 5$ pixels window around the aptamer positions for all frames and combined in intensity time traces. From these traces, on-times $\left(\tau_{o n}\right)$ and off-times $\left(\tau_{o f f}\right)$ were analyzed by setting a threshold of $3 \times$ the standard deviation above the mean background. For each time trace, on- and off-times were fitted with an exponential decay to obtain average $\tau_{\text {on }}$ and $\tau_{\text {off }}$ values at a single aptamer position. An error of $\pm 50 \mathrm{~ms}$ was assigned to these values to account for the camera dwell time. Average $\left\langle\tau_{o n}\right\rangle$ and $\left\langle\tau_{o f f}\right\rangle$ values taken over different aptamer positions were obtained (for identical TMR-DN concentration) by taking a weighted average according to the uncertainties of the average $\tau_{o n}$ and $\tau_{o f f}$ values of the individual aptamers. Average rate coefficients of dissociation $\left(k_{\mathrm{d}}=\left\langle T_{\mathrm{on}}\right\rangle^{-1}\right)$ and association $\left(k_{\mathrm{a}}^{\prime}=k_{\mathrm{a}} c=\left\langle T_{\mathrm{off}}\right\rangle^{-1}\right)$ were calculated. The final $k_{\mathrm{d}}$ was obtained by averaging over all concentrations and $k_{\mathrm{a}}$ by a linear regression to the concentration dependence (Fig. 3c). Data were taken at 1.1, 2.2, 4.5, 6.7 and 9.0 nM TMR-DN on 12 (410), 11 (610), 13 (1230), 8 (1000) and 10 (1730) individual molecules, respectively. The numbers in parentheses denote the total number of on-switching events.

SMLM imaging. A home-built widefield microscope based on an Axio Observer Z1 frame (Zeiss, Göttingen, Germany) with single-molecule sensitivity was used for SMLM, with small modifications from the design described earlier ${ }^{25}$. Light from a $473 \mathrm{~nm}$ laser (Gem 473, Laser Quantum, Konstanz, Germany) and a $561 \mathrm{~nm}$ laser (Gem 561, Laser Quantum) is combined via dichroic mirrors and passed through an AOTF (AOTFnC-400.650, A-A Opto-Electronic) to ensure precise and fast control of the laser intensities. Two achromatic lenses with focal lengths of 10 and $100 \mathrm{~mm}$ (Thorlabs) expand the beam after the AOTF. For widefield illumination, the beam is focused onto the back focal plane of a high numerical aperture oil 
immersion objective ( $\alpha$ Plan-Apochromat $63 \times / 1.46$ Oil Corr M27 TIRF, Zeiss) by a scan lens. Fluorescence emission is collected through the same objective and filtered by a quad-band dichroic mirror ( $z$ 405/473/561/640, AHF, Tübingen, Germany). After passing a tube lens, a beam splitter (OptoSplit II, Cairn Research, Kent, UK) separates the fluorescence into two images of different color, which are imaged side by side on an EMCCD camera (Ixon Ultra X-7759, Andor, Belfast, UK) with a pixel size of $109 \times 109 \mathrm{~nm}^{2}$.

Fixed DH5a bacteria expressing gfp (control), gfp-RhoBAST 16 , tolB-gfp-RhoBAST 16 , dsbA-gfpRhoBAST 16 and phoA-gfp-RhoBAST 16 were prepared and immobilized on a poly-D-lysine coated 8-well glass chamber as described before. Bacteria were incubated with $15 \mathrm{nM}$ TMR-DN in M9 medium for 30 min and imaged at room temperature. For the measurements, GFP was excited with the $473 \mathrm{~nm}$ laser; its emission was collected through a 525/45 nm (center/width) filter. 300 camera frames were collected using continuous illumination with an exposure time of $100 \mathrm{~ms}$ per frame and a laser power of $50 \mu \mathrm{W}$. Then, the excitation was swiftly changed to $561 \mathrm{~nm}$ by means of the AOTF, and TMR-DN was imaged using a 607/70 nm emission filter. 5,000 - 10,000 frames were collected with an exposure time of $100 \mathrm{~ms}$ each. The laser power was adjusted in the range $4.9-20.4 \mathrm{~mW}$ to achieve temporally well-separated blinking events in different cell regions.

To reduce noise in the GFP channel, 300 camera frames were averaged by using Fij/lmageJ software. For registration of the SMLM data in the TMR-DN channel, custom-written a-livePALM software was used $^{10}$. The major steps of the algorithm include background estimation and Gaussian noise filtering. Based on the detected background information, regions with local maxima are identified; their location is precisely determined with the maximum likelihood estimator (MLE) algorithm ${ }^{26}$. Localization accuracies of the SMLM images are calculated as described in Ref ${ }^{27}$.

Sample drift during data acquisition was corrected by a cross-correlation based analysis ${ }^{28}$. A substack of 500 frames was selected to reconstruct a reference image. The following sub-stack was reconstructed and its drift with respect to the reference frame was determined by cross-correlation and compensated. The combined image was subsequently taken as the new reference image. This procedure was repeated for all sub-stacks of the total image stack. 
SMLM imaging of mammalian cells. HEK293T cells expressing Tornado-RhoBAST were prepared as described before. For imaging live cells, $48 \mathrm{~h}$ after the transfection, the medium was exchanged by L15 medium containing $100 \mathrm{nM}$ TMR-DN. After incubation for $1 \mathrm{~h}$, cells were imaged at $37^{\circ} \mathrm{C}$ and $5 \% \mathrm{CO}_{2}$ in a live-cell imaging chamber. TMR-DN was imaged with $561 \mathrm{~nm}$ laser excitation $(3.1 \mathrm{~mW})$, the fluorescence was detected through a $607 / 70 \mathrm{~nm}$ emission filter. 1000 frames were collected with an exposure time of $30 \mathrm{~ms}$.

For imaging fixed cells, the cells were incubated in DPBS medium containing either $10 \mathrm{nM}$ or 20 $\mathrm{nM}$ TMR-DN and $1 \mathrm{mM} \mathrm{MgCl} 2$. After incubation for $1 \mathrm{~h}$, cells were imaged at room temperature with 561 $\mathrm{nm}$ excitation and a 607/70 $\mathrm{nm}$ emission filter. 5000 frames were collected with camera dwell times between 15 and $50 \mathrm{~ms}$ and laser powers between $3.1 \mathrm{~mW}$ and $14.6 \mathrm{~mW}$ to achieve well separated blinking events in each case.

The data were analyzed with the a-livePALM software ${ }^{10}$ as described above; no drift correction was applied. The final images were prepared with Fiji/imageJ; intensity profiles were analysed with Fiji/imageJ using a line width of five pixels at corresponding positions in the epifluorescence and SMLM images.

\section{References}

21. Strack, R.L., Disney, M.D. \& Jaffrey, S.R. A superfolding Spinach2 reveals the dynamic nature of trinucleotide repeat-containing RNA. Nat Methods 10, 1219-1224 (2013).

22. Grimm, J.B. et al. A general method to fine-tune fluorophores for live-cell and in vivo imaging. Nat Methods 14, 987-994 (2017).

23. Bajar, B.T. et al. Improving brightness and photostability of green and red fluorescent proteins for live cell imaging and FRET reporting. Sci Rep 6, 20889 (2016).

24. Manz, C. et al. Single-molecule FRET reveals the energy landscape of the full-length SAM-I riboswitch. Nat Chem Biol 13, 1172-1178 (2017).

25. Li, Y., Shang, L. \& Nienhaus, G.U. Super-resolution imaging-based single particle tracking reveals dynamics of nanoparticle internalization by live cells. Nanoscale 8, 7423-7429 (2016).

26. Ober, R.J., Ram, S. \& Ward, E.S. Localization accuracy in single-molecule microscopy. Biophys J 86, 1185-1200 (2004).

27. Deschout, H. et al. Precisely and accurately localizing single emitters in fluorescence microscopy. Nat Methods 11, 253-266 (2014).

28. Guizar-Sicairos, M., Thurman, S.T. \& Fienup, J.R. Efficient subpixel image registration algorithms. Opt Lett 33, 156-158 (2008). 


\section{Acknowledgements}

M.S. and A.J. were supported by the Deutsche Forschungsgemeinschaft (DFG Grant \# Ja794/11-1) and G.U.N. by the Helmholtz Association (Program Science and Technology of Nanosystems) and by the DFG (GRK 2039). We thank the Nikon Imaging Center, Heidelberg for granting access to their facilities and Dr. Ulrike Engel for technical advice in fluorescence microscopy.

\section{Author contributions}

M.S., G.U.N. and A.J. designed the study. A.M. and M.S. evolved RhoBAST. D.E. and M.S. characterized RhoBAST. M.S. created all plasmid constructs and strains. M.S carried out confocal and SIM microscopy. J.L. and B.H. established single-molecule binding kinetics experiments. J.L. carried out SMLM. M.S., K.N., G.U.N. and A.J. supervised the work. M.S. wrote the first draft and all authors contributed to reviewing, editing and providing additional text for the manuscript.

\section{Competing interests}

The authors declare no competing interests.

\section{Additional information}

Supplementary information is available for this paper.

Correspondence and request for materials should be addressed to M.S. or A.J. 